\title{
Análise sumária de urina de rotina: porquê e para quê?
}

Carla Lopes da Mota,* Helena Paula Beça**

\section{RESUMO}

Objectivo: Determinar qual o benefício da análise sumária de urina no rastreio de adultos assintomáticos, à luz da melhor evidência disponível.

Fontes de dados: Cochrane Library, Trip Database, Dare, National Guideline Clearinghouse, Finland Evidence Based Medicine Guidelines, PubMed, UpToDate, Index de Revistas Médicas Portuguesas e nas citações relacionadas.

Métodos de revisão: Foi realizada uma pesquisa nas bases de dados citadas, utilizando os termos MeSH urinalysis e mass screening. A pesquisa foi limitada aos artigos publicados até Março de 2012, em inglês e português. Para avaliar o nível de evidência e a força de recomendação foi utilizada a taxonomia SORT (Strength of Recommendation Taxonomy) da American Academy of Family Physicians.

Resultados: Foram encontrados 459 artigos e seleccionados, por cumprirem os critérios de inclusão, uma revisão sistemática, dois artigos originais, duas normas de orientação clínica e um artigo de revisão. Após a sua análise, verifica-se que, apesar de ser um exame frequentemente pedido, a alteração à orientação clínica previamente definida ocorre em menos de $5 \%$ dos casos, implicando nestes a realização de novos exames complementares de diagnóstico mais onerosos e invasivos sem benefício relevante para a maioria dos utentes.

Conclusões: O uso da análise sumária de urina, como teste de rastreio em adultos assintomáticos, não demonstrou benefício, pelo que não está recomendado (SOR A). A evidência contra o seu uso sistemático é clara, consistente e tem já várias décadas, como comprovado pela data de realização dos estudos apresentados e pela inexistência de estudos recentes. No entanto, em Portugal mantém-se a realização deste teste nas mais diferentes circunstâncias, como "rotina" ou estudo pré-operatório, pelo que se sugere a definição de novas regras na sua utilização.

Palavras-chave: Análise Sumária de Urina; Rastreio.

\section{INTRODUÇÃO}

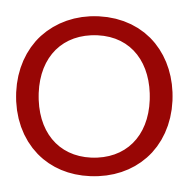

pedido de análise sumária de urina é um acto rotineiro, realizado diária e repetidamente. O seu uso visa o rastreio de bacteriúria assintomática, hematúria e/ou proteinúria e inclui o exame físico, químico e microscópico da urina. São vários os parâmetros analisados, como a coloração, a densidade, o pH, a hematúria, proteinúria, leucocitúria e glicosúria, entre outros. As diferentes alterações no exame poderão ser atribuídas ao uso de fármacos e a inúmeras patologias, entre as quais a diabetes mellitus, a tuberculose, a doença renal (glo-

*Assistente de Medicina Geral e Familiar - UCSP Barão do Corvo II (Afurada) - ACeS Grande Porto VII - Gaia

**Assistente de Medicina Geral e Familiar - USF Espinho - ACeS Grande Porto VIII - Espinho/Gaia merulopatias) e a neoplasia e as doenças infecciosas do aparelho urinário (cistite, pielonefrite). ${ }^{1}$ Apesar do seu baixo custo, o número de exames efectuados anualmente pode ter um impacto significativo nos encargos associados à saúde e na qualidade de vida dos indivíduos, porque os frequentes falsos positivos acarretam investigação adicional, o que aumenta os custos e implica riscos para o doente.

A Confederação Europeia de Medicina Laboratorial, através do European Urinalysis Group, publicou em 2000 uma guideline na qual estabelece que a análise sumária de urina deve ser apenas solicitada por indicação clínica. ${ }^{2} \mathrm{O}$ seu uso sistemático em determinadas populações deve ser sempre analisado numa perspectiva custo/benefício. ${ }^{2}$ Poderá ser discutível, por exemplo, nos doentes hipertensos ou diabéticos, já que actualmente a determinação da microalbuminúria é o 
parâmetro mais importante e esta poderá ser efectuada recorrendo a uma determinação específica (doseamento da razão albuminúria/proteinúria). ${ }^{3-4}$ Também, nos indivíduos com factores de risco para a neoplasia da bexiga (fumadores, exposição a carcinogéneos químicos, entre outras condições) e, de acordo com a U.S. Preventive Services Task Force, parece não existir evidência de diminuição da mortalidade e/ou morbilidade com o rastreio através da análise sumária de urina, pelo que este não é recomendado. ${ }^{5}$

Perante estes dados, qual será então o benefício da realização da análise sumária de urina, de forma sistemática, em adultos aparentemente saudáveis e sem factores de risco, no que se refere à diminuição de morbi-mortalidade e melhoria da qualidade de vida?

Com este trabalho pretendeu-se determinar qual o benefício da análise sumária de urina no rastreio de adultos assintomáticos, à luz da melhor evidência disponível.

\section{MÉTODOS}

Foi realizada uma pesquisa bibliográfica de meta-análises, revisões sistemáticas, ensaios clínicos aleatorizados e controlados, normas de orientação clínica e artigos de revisão, nas bases de dados Cochrane $L i$ brary, Trip Database, Dare, National Guideline Clearinghouse, Finland Evidence Based Medicine Guidelines, PubMed, UpToDate, Index de Revistas Médicas Portuguesas e nas citações dos artigos encontrados, utilizando os termos MeSH urinalysis e mass screening. A pesquisa foi limitada aos artigos publicados até Março de 2012 em inglês e português. Para avaliar o nível de evidência e estabelecer a força de recomendação foi utilizada a taxonomia SORT (Strength of Recommendation Taxonomy) da American Academy of Family Physicians. ${ }^{6}$

Foram seleccionados os artigos que incluíam indivíduos adultos, assintomáticos, que tinham realizado a análise sumária de urina sem indicação clínica. Os outcomes avaliados foram a percentagem de análises anormais, alteração na conduta clínica (acarretando mais exames, por vezes invasivos), efeitos adversos para o doente e diagnóstico precoce com melhoria da morbimortalidade. Foram excluídos os artigos repetidos ou que não cumpriam os critérios de inclusão no que se refere à população, comparação ou outcomes.

\section{RESULTADOS}

Foram encontrados 459 artigos e seleccionados, por cumprirem os critérios de inclusão, uma revisão sistemática, dois artigos originais, duas normas de orientação clínica e um artigo de revisão (Quadro I).

A revisão sistemática, de Munro Jetal (1997), incluiu 11 estudos. Em sete destes a amostra era constituída por indivíduos adultos, sendo que em todos foi analisada a percentagem de resultados anormais, em cinco a alteração da conduta clínica e em dois os efeitos adversos para os indivíduos com resultados anormais. Dois trabalhos foram realizados na admissão a um serviço de urgência de medicina e os restantes em condições pré-operatórias. De uma forma global foram realizados 6740 testes, sendo apresentados os resultados no quadro I. Analisando-os, verifica-se que em cerca de um terço dos casos foram encontrados resultados anormais, sendo que a grande maioria destes foi ignorada pelos médicos, uma vez que a alteração na conduta clínica foi rara $(0,1 \%$ a $2,8 \%)$. Esta diferença, entre o número de resultados anormais e as intervenções subsequentes, poderá estar relacionada com a heterogeneidade da população estudada, assim como com as possíveis classificações de resultados anormais utilizadas. Outra explicação poderá ser a importância dúbia atribuída pelos clínicos a este exame como rastreio de doença. Nenhum dos estudos demonstrou qualquer evidência de que uma análise sumária de urina anormal, pré-operatória, esteja associada com algum evento adverso peri ou pós-cirurgia, não sendo preditivo de complicações. ${ }^{7}$ Apesar de não ser objecto desta revisão, destaca-se o facto de ausência de benefício parecer ser independente da idade, de acordo com este trabalho.

Quanto aos artigos originais, Ruttimann S et al (1994) comparou a realização da análise sumária de urina com e sem indicação clínica, na admissão a uma clínica de Medicina Interna (que incluía actividades preventivas e serviço de urgência). Consultando o quadro I, verifica-se que, em 427 doentes, apenas $0,7 \%$ foram submetidos a alteração da conduta clínica (3 doentes). ${ }^{8}$ No segundo artigo original, Pashayan N et al (2002) realizou um estudo retrospectivo referente ao uso da análise sumária de urina com e sem indicação clínica na admissão a um serviço de Medicina e Cirurgia no Líbano, onde as actividades preventivas estão sub-desenvolvidas e a prevalência de doença renal parece ser diferen- 
te da registada no ocidente, de acordo com os autores. Participaram 462 doentes, tendo sido alterada a conduta clínica em apenas 2 doentes que realizaram o exame como rastreio e em 26 que o fizeram com indicação clínica. Comparando com a realização por rastreio, a análise sumária de urina efectuada por suspeição clínica apresentava uma probabilidade duas vezes superior de ser anormal, três vezes maior dessa anomalia ser investigada e 19 vezes superior de produzir um novo diagnóstico. ${ }^{9}$

No que diz respeito às normas de orientação clínica, as Finland Evidence Based Medicine Guidelines (2010) estabelecem que a análise sumária de urina deve ser um teste considerado de forma individual, de acordo com a indicação clínica, com uma força de recomendação A. ${ }^{10} \mathrm{O}$ Institute for Clinical Systems Improvement (2010)

\begin{tabular}{|c|c|c|c|c|c|c|}
\hline Autor, ano & $\begin{array}{l}\text { Tipo de } \\
\text { estudo }\end{array}$ & \multicolumn{2}{|l|}{$\begin{array}{l}\text { Exposição/ } \\
\text { /Comparação }\end{array}$} & Outcomes & Resultados & NE/FR \\
\hline $\begin{array}{l}\text { Munro J, } \\
\text { et al, } \\
1997\end{array}$ & $\begin{array}{l}\text { Revisão } \\
\text { sistemática } \\
11 \text { estudos } \\
(\mathrm{n}=147 \text { a } \\
3987)\end{array}$ & \multicolumn{2}{|c|}{$\begin{array}{l}\text { Realização de análise } \\
\text { sumária de urina (ASU) } \\
\text { no pré-operatório }\end{array}$} & $\begin{array}{l}\text { Percentagem de resultados } \\
\text { anormais e de resultados } \\
\text { anormais clinicamente } \\
\text { significativos } \\
\text { Alteração da conduta clínica } \\
\text { Incidência de efeitos adversos } \\
\text { nos doentes com teste anormal }\end{array}$ & $\begin{array}{l}\text { Resultados anormais em } \\
1 \text { a } 34,1 \% \text { dos doentes } \\
\text { Alteração da conduta } \\
\text { clínica em } 0,1 \text { a } 2,8 \% \\
\text { dos doentes } \\
\text { Efeitos adversos em } \\
0-0,6 \% \text { dos doentes com } \\
\text { teste anormal }\end{array}$ & 1 \\
\hline $\begin{array}{l}\text { Ruttiman } \\
\text { S, et al, } \\
1994\end{array}$ & $\begin{array}{l}\text { Estudo coorte } \\
\text { prospectivo } \\
(n=610)\end{array}$ & \multicolumn{2}{|c|}{$\begin{array}{l}\text { Realização ASU com } \\
\text { indicação clínica versus } \\
\text { sem indicação clínica }\end{array}$} & $\begin{array}{l}\text { Alteração da conduta clínica } \\
\text { Percentagem de resultados } \\
\text { anormais }\end{array}$ & $\begin{array}{l}\text { ASU sem indicação } \\
\text { clínica em } 70 \% \text { dos } \\
\text { casos } \\
\text { Resultados anormais em } \\
17 \% \\
\text { Alteração da conduta } \\
\text { clínica em } 0,7 \% \text { dos } \\
\text { doentes }\end{array}$ & 2 \\
\hline $\begin{array}{l}\text { Pashayan } \\
\mathrm{N}, \text { et al, } \\
2002\end{array}$ & $\begin{array}{l}\text { Estudo coorte } \\
\text { retrospectivo } \\
(n=462)\end{array}$ & \multicolumn{2}{|c|}{$\begin{array}{l}\text { Realização de ASU com } \\
\text { indicação clínica versus } \\
\text { sem indicação clínica }\end{array}$} & $\begin{array}{l}\text { Frequência de ASU sem } \\
\text { indicação clínica } \\
\text { Investigação de resultados } \\
\text { anormais } \\
\text { Alteração na conduta clínica }\end{array}$ & $\begin{array}{l}\text { ASU sem indicação } \\
\text { clínica em } 70 \% \text { dos casos } \\
37 \% \text { resultados anormais, } \\
\text { apenas } 22 \% \text { investigados } \\
\text { Alteração terapêutica } \\
\text { em } 1 \%\end{array}$ & 2 \\
\hline \multicolumn{2}{|l|}{ Autor, ano } & Tipo de estudo & \multicolumn{3}{|c|}{ Recomendação } & NE/FR \\
\hline \multicolumn{2}{|c|}{$\begin{array}{l}\text { Finland Evidence Based } \\
\text { Medicine Guidelines, }{ }^{10} 2010\end{array}$} & $\begin{array}{l}\text { Norma de } \\
\text { Orientação Clínica }\end{array}$ & \multicolumn{3}{|c|}{$\begin{array}{l}\text { A ASU deve ser considerada de forma individual de acordo com a } \\
\text { indicação clínica }\end{array}$} & A \\
\hline \multicolumn{2}{|c|}{$\begin{array}{l}\text { Institute for Clinical Systems } \\
\text { Improvement, }{ }^{11} 2010\end{array}$} & $\begin{array}{l}\text { Norma de } \\
\text { Orientação Clínica }\end{array}$ & \multicolumn{3}{|c|}{ Contra o pedido de ASU sem indicação clínica } & A \\
\hline \multicolumn{2}{|c|}{ Kiel D, et al, ${ }^{12} 1987$} & $\begin{array}{l}\text { Artigo de revisão } \\
\text { não sistemática }\end{array}$ & \multicolumn{3}{|c|}{$\begin{array}{l}\text { Não existe evidência na literatura que suporte o pedido de ASU } \\
\text { em indivíduos assintomáticos }\end{array}$} & 3 \\
\hline
\end{tabular}

Legenda: ASU - análise sumária de urina; FR - força de recomendação; NE - nível de evidência. 
considera que não existe evidência que suporte o uso da análise sumária de urina sem indicação clínica, emitindo uma recomendação contra o seu pedido como teste de rastreio (força de recomendação A). ${ }^{11}$

O artigo de revisão incluído, de Kiel Detal (1987), refere que não existe evidência na literatura que suporte o pedido de análise sumária de urina em indivíduos assintomáticos (Nível de evidência 3). ${ }^{12}$

\section{CONCLUSÕES}

A análise sumária de urina é um teste simples, não invasivo e pouco dispendioso, o que pode contribuir para a sua realização de forma rotineira e sem critério clínico e para explicar o reduzido investimento em estudos recentes que determinem o benefício real deste teste em indivíduos assintomáticos. No entanto, após a análise dos resultados obtidos nesta revisão, a evidência contra o uso sistemático da análise sumária de urina está claramente demonstrada em estudos realizados há várias décadas. Foi também perceptível o baixo valor que os clínicos atribuem a este exame, ignorando na grande maioria os seus resultados, independente de anormais ou não.

No entanto, são ainda necessários mais estudos para clarificar se o uso da análise sumária de urina diminui a morbi-mortalidade em populações seleccionadas, como os diabéticos ou os hipertensos, assim como determinar se a ausência de benefício é independente da idade, como parece demonstrar a literatura. ${ }^{7}$

Como em Portugal persiste a sua realização, muitas vezes sem critério clínico adequado, sugere-se a definição de novas orientações no seu pedido.

Em conclusão, o uso da análise sumária de urina como teste de rastreio em adultos assintomáticos não traz qualquer benefício, pelo que não está recomendado (SOR A).

\section{REFERÊNCIAS BIBLIOGRÁFICAS}

1. Simerville JA, MaxtedWC, Pahira JJ. Urinalysis: a comprehensive review. Am Fam Physician 2005 Mar 15; 71 (6): 1153-62.

2. European Confederation of Laboratory Medicine. European urinalysis guidelines. Scand J Clin Lab Invest Suppl 2000; 231: 1-86.

3. Direção-Geral de Saúde. Norma de Orientação Técnica n 037/2011: Exames laboratoriais na gravidez de baixo risco. Lisboa: DGS; 2011.

4. Direção-Geral de Saúde. Norma de Orientação Técnica nº 08/2011: Diagnóstico sistemático de nefropatia diabética. Lisboa: DGS; 2011.

5. U.S. Preventive Services Task Force. Guide to Clinical Preventive Services. Rockville, MD:Agency for Healthcare Research and Quality; 2012.

6. Ebell MH, Siwek J, Weiss BD, Woolf SH, Susman J, Ewigman B, et al. Strength of recommendation taxonomy (SORT): a patient-centered approach to grading evidence in the medical literature. Am Fam Physician 2004 Feb 1; 69 (3): 548-56.

7. Munro J, Booth A, Nicholl J. Routine preoperative testing: a systematic review of the evidence. Health Technol Assess 1997; 1 (12): 1-62.

8. Rüttimann $S$, Clémençon $D$. Usefulness of routine urine analysis in medical oupatients. J Med Screen 1994 Apr; 1 (2): 84-7.

9. Pashayan N, Khogali M, Major SC. Routine urinalysis of patients in hospital in Lebanon: how worthwihle is it? J Med Screen 2002; 9 (4): 1816.

10. Finland Evidence Based Medicine Guidelines. Urinalysis and bacterial culture. 2010. Disponível em: http://ebmg.onlinelibrary.wiley.com/ ebmg/ ltk.koti [acedido em 01/03/2013].

11. Institute for Clinical Systems Improvement (ICSI). Preventive Service for Adults. Bloomington (MN): ICSI; 2010

12. Kiel DP, Moskowitz MA. The urinalysis: a critical appraisal. Med Clin North Am 1987 Jul; 71 (4): 607-24.

\section{CONFLITO DE INTERESSES}

As autoras são editoras da Revista Portuguesa de Medicina Geral e Familiar e não participaram no processo de edição deste artigo.

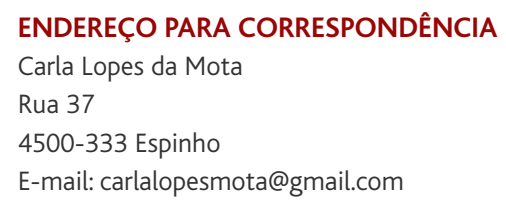

Recebido em 18/06/2012

Aceite para publicação em 08/02/2013 


\section{ABSTRACT}

\section{ROUTINE URINALYSIS: WHY AND WHEN?}

Objectives: To determine the benefit of urinalysis in screening asymptomatic adults from the best evidence available. Data Sources: Cochrane Library, Trip Database, Dare, National Guideline Clearinghouse, Finland Evidence Based Medicine Guidelines, PubMed, UpToDate, Index of Medical Portuguese Journals and related citations.

Review Methods: Search for articles and related citations using the MeSH terms urinalysis and mass screening, published until March of 2012, in English and Portuguese. The Strength of Recommendation Taxonomy (SORT) scale of the American Academy of Family Physicians was used for assigning levels of evidence and the strength of recommendation.

Results: The search produced a total of 459 articles. Six met the inclusion criteria. These included one systematic review, two original studies, two clinical practice guidelines, and one review article. Although urinalysis is frequently ordered, a change in management occurs in less than $5 \%$ of the cases after the test. It may lead to the ordering of other more expensive and invasive diagnostic tests.

Conclusions: Urinalysis as screening test in asymptomatic adults is not beneficial for patients. It is not recommended as a screening test (SOR A). The evidence against systematic use has been clear for several decades. In Portugal it is commonly requested as a «routine» or preoperative test. In the light of published evidence, new guidelines seem necessary.

Keywords: Urinalysis; Screening. 